\title{
Movement Aftereffect of Bi-Vectorial Transparent Motion
}

\author{
FRANS A. J. VERSTRATEN,** R. ERIC FREDERICKSEN, † WIM A. VAN DE GRIND* \\ Received 30 September 1992; in revised form 1 April 1993; in final form 18 June 1993
}

\begin{abstract}
Two moving random-pixel arrays (RPAs) were presented simultaneously in the same target field. These RPAs are perceived as two superimposed transparent moving sheets. Although two directions are perceived simultaneously during stimulus presentation, the movement aftereffect (MAE) is unidirectional. The visual system averages both motion signals in the MAE. For motion vectors of equal magnitude and perpendicular direction the MAE direction is the inverse of the sum of both vectors. In the first experiment we measured perceived direction of the MAE of transparent motion for a range of speed combinations. Results indicate that vector summation only predicts the correct MAE direction for combinations of equal speeds. It is suggested that the direction of the MAE of transparent motion is a resultant of the weighted summation of the component inducing vectors. The question then arises what determines the weighting factors. Directional sensitivity and MAE duration of the individual vectors under transparent conditions were measured and used to weigh the vectors and predict the MAE direction of transparent motion. Statistical analyses showed that MAE duration is a better basis to determine the weighting factors predicting the direction of the MAE of transparent motion than component sensitivity. The direction of the MAE of transparent motion thus seems to be determined by the amount of adaptation to the component vectors as reflected by MAE duration. The results suggest that this gain control cannot be located in the individual motion detectors and must be situated at or after some subsequent cooperation stage of the human motion analysis system.
\end{abstract}

Aftereffects Transparent motion Adaptation MAE duration Gain control

\section{INTRODUCTION}

If you look at a far point in a landscape from a window seat of a moving train it is often possible to see bushes and rows of trees at different distances move transparently "through" each other. This means that it is possible to discriminate several simultaneously present moving textures in the same region of the visual field. The textures obviously need holes through which one gets glimpses of the other textures, and these other layers need to move in sufficiently different directions and/or at sufficiently different velocities. Clarke (1977) reported a simulated version of this natural transparent motion phenomenon. He quickly alternated two random-dot patterns moving in opposite directions. At low alternation rates, the percept was that of a single noise pattern that regularly reversed its direction. When the alternation frequency exceeded about $12 \mathrm{~Hz}$, dynamic noise was perceived. For alternation frequencies higher than approx. $40 \mathrm{~Hz}$ transparent motion was perceived. These

*Utrecht Biophysics Research Institute, Department of Comparative Physiology, Utrecht University, Padualaan 8, NL-3584 CH Utrecht, The Netherlands.

†Department of Computer Science, University of North Carolina, Chapel Hill, NC 27599-3175, U.S.A.

$\ddagger$ Present address: McGill Vision Research Centre, 687 Pine Avenue West (H4, 14), Montreal, Canada H3I IAl. findings were confirmed by van Doorn and Koenderink (1982a) who alternated periodically between two moving random-pixel arrays with velocity vectors $V_{1}$ and $V_{2}$. At a sufficiently fast alternation rate both patterns were seen to move in transparent fashion, each with its own velocity and motion direction (if the directions were sufficiently different). They also described a spatial variant of this phenomenon (van Doorn \& Koenderink, $1982 b$ ) in which a moving random-pixel array A was present in "even" windows and B in "odd" windows on a screen divided up into many stripe-like contiguous windows. This "spatial" transparency requires relatively narrow stripes and is reminiscent of the natural case with a row of trees in the foreground, bushes in the background and a fixation in between the two layers. There are other ways to mimic natural motion transparency, e.g. as in this paper with a checkerboard pattern of contiguous windows displaying the moving $A$ and $B$ patterns.

A surprising finding was reported by van Doorn et al. (e.g. van Doorn, Koenderink \& van de Grind, 1984, 1985), who found that the motion after-effect (MAE) of transparent motion is a single unified MAE. Using a different technique Mather (1980) also reported a unidirectional MAE after adaptation to a two-dimensional pattern. Thus two vectors describe the perceived motions during the adaptation period but only a single vector is required to characterize the resulting MAE. 
This means that some fusional combination of internal signals takes place either during the adaptation phase (even though no combined motion is perceived) or during the MAE test phase. The result is a kind of vector averaging, where the adaptation vectors $V_{1}$ and $V_{2}$ can be said to induce a MAE vector $V_{a}$

$$
\mathbf{V}_{\mathrm{a}}=c_{1} \mathbf{V}_{1}+c_{2} \mathbf{V}_{2}
$$

Van Doorn et al. found that (1), with $c_{1}=c_{2}=c(c<0)$ approximately describes the direction of the MAE for the case of two adaptation component movements of about equal magnitude $\left(\left|\mathbf{V}_{1}\right| \approx\left|\mathbf{V}_{2}\right|\right)$. The proposal of formula (1) has not yet been checked for different magnitudes of the motion vectors. It is the purpose of this paper to quantify this "averaging" operation in more detail and to relate the results to existing models of movement aftereffects.

\section{Frameworks for reasoning about the $M A E$}

Normally, after a period of adaptation to a pattern moving in a certain direction, a subsequently viewed stationary pattern appears to move in the opposite direction (e.g. Wohlgemuth, 1911). This aftereffect is often explained in terms of the ratios of firing in cells sensitive to movement in opposite directions (Sutherland, 1961; Barlow \& Hill, 1963). The motion in a certain direction fatigues groups of neurons (detectors) tuned to that direction. When presented with a stationary pattern the neurons which had just been stimulated will fire at a reduced rate (below spontaneous activity). The ratio of firing or balance between the oppositely tuned detectors is unequal and hence an illusionary movement in the opposite direction is perceived. This "classical" explanation obviously requires some push-pull combination of oppositely directed motion detectors.

The same reasoning cannot be used to explain the direction of the MAE of transparent motion. As was already noted by Mather (1980), the "classical" model would predict two simultaneously present MAEs opposite to the two adapting directions. Mather proposed an alternative model, called the "distribution shift model" (Mather, 1980; Mather \& Moulden, 1980). In contradistinction to Sutherland's ratio model, in which only the activity of oppositely tuned motion detectors is compared, the distribution shift model takes the activity of all motion detectors with all direction preferences into account. For example, two transparent vectors like we used would fatigue detectors tuned to the directions towards 1.30 o'clock (45 deg-vector 1) and towards 10.30 o'clock (135 deg-vector 2) and (to a lesser extent) detectors tuned to directions close to, that is differing less than the tuning width from, the adaptation directions. So if a stationary pattern is presented neurons tuned to those directions fire less. Taking all activity into account, the activity maximum for the pool would be directed toward 6 o'clock $(270 \mathrm{deg})$ if the magnitudes (speeds) of both adaptation vectors are the same.

This model implies that the direction of the MAE of transparent motion is a weighted resultant of the component vectors of the inducing stimulus. What is weighted and how still has to be quantified, however, at least for the case of transparent motion. In this study we determine the direction of the MAE of transparent motion for several speed combinations. The results show that formula (1) indeed describes the case for equal speeds but is incorrect for other combinations. An obvious refinement would be to replace the constants by functions, e.g. of the speeds. If something like formula (1) would hold for the internal (neural) representations of $\mathbf{V}_{1}$ and $\mathbf{V}_{2}$ for example, we would expect the weighting factors of these two representations to influence their vector summation. Thus $c_{1}$ and $c_{2}$ might have to be replaced by motion sensitivity functions. We wanted to test this idea and thus measured directional motion sensitivity and aftereffect duration for the individual vectors under transparent conditions. It is shown that the "internal" vector summation proposal leads to a good description of our results. MAE duration, however, is a better predictor of component weight than directional sensitivity. This is related to MAE models in the Discussion.

\section{GENERAL METHODS}

In all experiments reported in this paper we used random-pixel arrays (RPAs). These RPAs were generated by a specially designed noise generator controlled by a Macintosh IIfx (for a more detailed description see Fredericksen, Verstraten \& van de Grind, 1993) and were presented on a CRT display (ElectroHome model EVM-1200, P4 phosphor). The refresh rate of the display was $90 \mathrm{~Hz}$. The display contained $256 \times 256$ pixels and was $14 \mathrm{~cm}$ square ( 1 pixel $\approx 0.55 \mathrm{~mm}$ ). Viewing distance was $2 \mathrm{~m}$. The display area subtended $4 \mathrm{deg}$ arc. Each pixel subtended an angle of $0.94 \mathrm{~min}$ arc. Mean luminance of the CRT display was $50 \mathrm{~cd} \mathrm{~m}^{-2}$

Transparent motion was established by spatial transparency (see van Doorn \& Koenderink, 1982b). However, we used a different version called spatial "checkerboard" transparency: a checkerboard pattern of contiguous windows $(1 * 1$ pixels) displaying the moving $\mathrm{A}$ and $\mathrm{B}$ patterns. The main advantage of this checkerboard principle is the possibility to use smaller speed steps, at least in the present case where the direction of the vectors is $45 \mathrm{deg}$ to either side of vertically upwards.

In all experiments the same three male subjects participated, FV, WD and JH. Two subjects were myopic [1 D (FV) and 4.5 D (WD)]. Vision was corrected to normal. Subjects WD and JH were naive as to the hypothesis until the experiments were finished. A chin rest and forehead support were used. In all experiments the subjects viewed the display foveally and monocularly (FV, right eye; WD, left eye; JH, right eye). A black fixation dot (dia approx. $3 \mathrm{~min}$ arc) was present in the centre of the field. The experiments were performed in a dark room with ambient lighting provided by the display screen. 


\section{EXPERIMENT 1}

Van Doorn et al. (1984) suggested that for equal speeds the direction of the MAE of transparent motion is opposite to the vector sum of the adaptation components. In the following experiment we present motion vectors under transparent conditions with equal as well as unequal speeds. After adaptation subjects have to indicate the direction of the integrated MAE.

\section{Methods and procedure}

The subjects were presented with transparent motion for $60 \mathrm{sec}$ (adaptation phase). For the next $10 \mathrm{sec}$ a stationary random-dot pattern with an adjustable line (a black line of 1 pixel width projected on the pattern) was visible. The orientation of the line could be adjusted in either steps of 10 or $1 \mathrm{deg}$ by using the computer keyboard. The subject's task was to adjust the orientation of the line such that it was parallel to the direction of the MAE (adjust phase). These $10 \mathrm{sec}$ were followed by $45 \mathrm{sec}$ of top-up adaptation. A stationary pattern was again presented and the subject could check whether the line was in the right orientation. If not, the line orientation could be adjusted again. The alternation between top-up adaptation and adjustment was repeated until the subject was satisfied that the line was parallel to the direction of the MAE. Subjects were instructed to fixate during the whole experiment except for the pauses. The computer registered the line orientation after every adjust phase. After the subject indicated that the line was in a satisfactory orientation a pause of $90 \mathrm{sec}$ was given before the next trial was started.

The direction of the inducing vectors was always the same, vector 1 from the bottom-left to the top-right position (45 deg-bltr direction) and vector 2 from the bottom-right to the top-left position $(135 \mathrm{deg}-$ brtl direction). All the combinations of available speeds (magnitudes) for vector 1 and vector 2 were presented where the available speeds were $0.25,0.5,1,2,4,6,8,10,12$, and $14 \mathrm{deg} \mathrm{sec}^{-1}$. This gave 100 combinations which were randomized over 13 sessions, each lasting about 35 min. Subject FV and JH did all combinations. Subject WD was presented with speeds $1,4,6,8 \mathrm{deg} \mathrm{sec}^{-1}$ for vector 2 combined with all speeds for vector 1 .

\section{Results and discussion}

Figure 1 presents the results of this first experiment for subject FV (the results for the other subjects are displayed in Fig. 5). The panels show the measured directions of the MAE as a function of speed 2 with speed 1 as a parameter.

We use the mathematical angle specification convention indicated at the bottom of panel "14" (see Fig. 1). The predicted direction for the MAE of transparent motion according formula (1) was calculated from the formula

$$
\alpha_{\mathrm{MAE}}=225 \mathrm{deg}+\arctan \left(\frac{V_{2}}{V_{1}}\right)
$$

where $V_{2}$ and $V_{1}$ denote the magnitudes of the corresponding velocity vectors. The solid lines in the panels of Fig. 1 represent this prediction. The open squares are the empirical results. Subjects needed 1-5 repeated measurements to make a decision. The results as presented in the graphs are the values of the final settings. Subject $\mathrm{JH}$ reported difficulties in setting the direction for combinations between low and high speeds. This is due to the absence or short duration of the MAE of transparent motion of either of its inducing

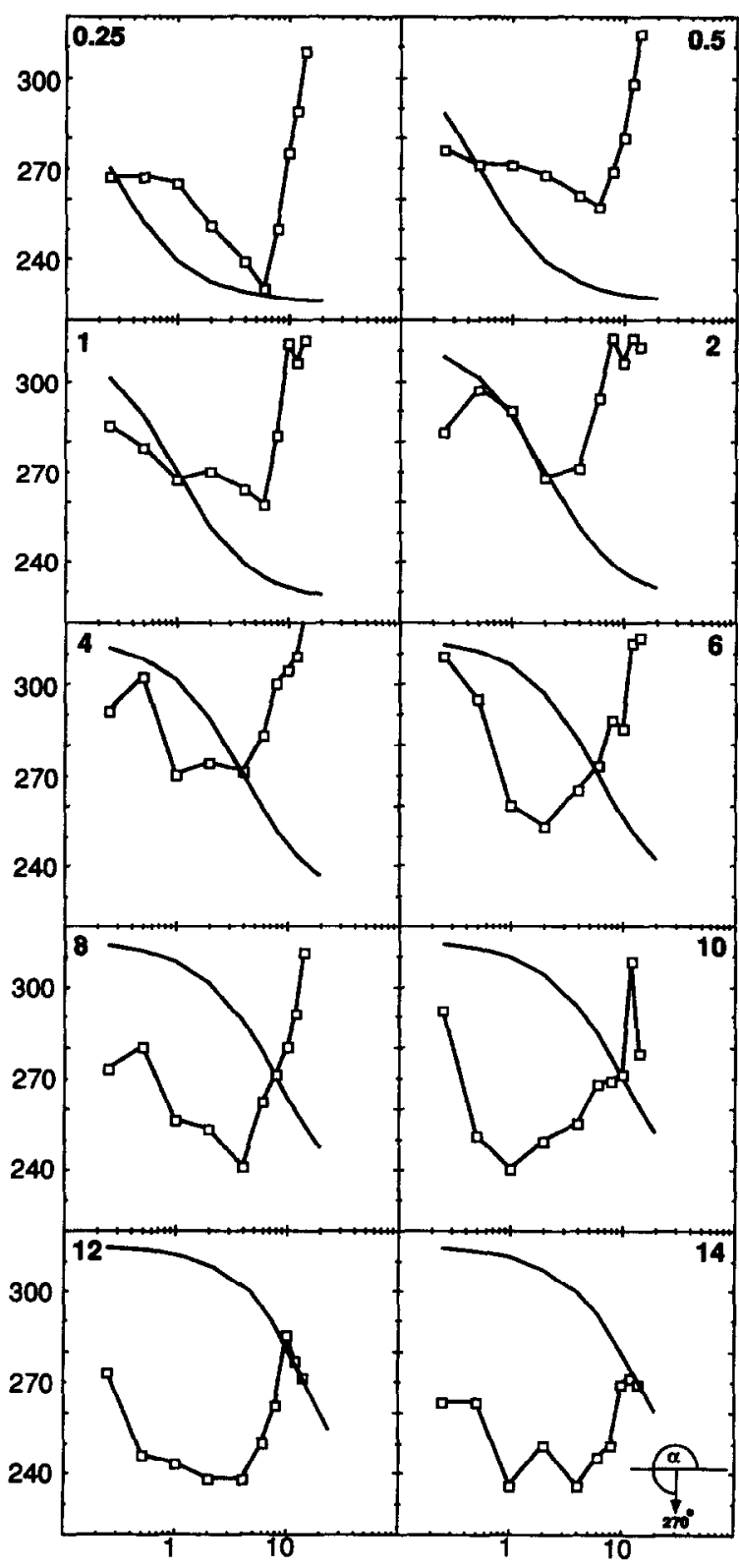

Pattern Speed (deg/s)

FIGURE 1. In these 10 panels the results from Expt 1 are displayed for subject FV. For ease of distinction the panels present the measured direction of the MAE as a function of $V_{2}$ with $V_{1}$ as a parameter. The MAE direction is in degrees (angle $\alpha$ is defined in the panel labelled "14"). Solid line without symbols represents the values as predicted on the basis of simple vector summation (see text). Open squares represent the values of the MAE direction for transparent motion as found in Expt 1 . Note that the empirical and the predicted direction are only (about) equal for combinations of equal speeds, $V_{1}=V_{2}$. 
components for this subject (this was found in Expt 2). When a proper MAE was perceived, it was unidirectional and no subject reported changes in the direction of the MAE during the test phase.

It is obvious from Fig. 1 that combinations of equal speeds result in an aftereffect directed downwards. Small variations may be due to anisotropies in the visual field or to small inaccuracies in setting the direction line. Each combination was recorded once. In order to get insight in the variance of the settings subject FV repeated several combinations three times. The variances varied from 3 to $12 \mathrm{deg}$ in angle. Variances were highest for combinations of low speeds.

The main message is clear; we are not dealing with a simple linear, speed based, vector summation system. The scalars $c_{1}$ and $c_{2}$ of formula (1) are obviously not constants. In the case $V_{1}=V_{2}$ the formula yields correct results if $c_{1}=c_{2}$. For other combinations of $V_{1}$ and $V_{2}$, $c_{1}$ and $c_{2}$ need to be adjusted and looking at the data presented in Fig. 1 it is tempting to suggest that the scalars $c_{1}$ and $c_{2}$ of formula (1) should be some function of the velocity. Our goal in the next experiment is to find out whether this is the case.

\section{EXPERIMENT 2}

\section{Rationale}

Can the direction of the MAE be accurately predicted if we take motion sensitivity to the individual motion components into account? It is known that our visual system is most sensitive to velocities of about 1-6 deg sec${ }^{-1}$ (e.g. Sekuler \& Pantle, 1967; Richards, 1971). Motion sensitivity can be viewed as a measure of the number of detectors available for a certain velocity in the tested area. The number of detectors for 1-6 deg sec$~^{-1}$ might thus be greater than for slower and faster speeds. These velocities are indeed very dominant in influencing the direction of the MAE of transparent motion. In the first part of Expl 2 we measure the motion sensitivity curve for all speeds that were used in Expt 1 under transparent conditions. Two patterns were visible, one was moving, the other pattern was stationary. Our assumption is that under these conditions the same number of motion detectors for the remaining one direction is stimulated as in the condition where both component patterns are moving.

Another psychophysical correlate that seems to have approximately the same dependence on the stimulus parameter as sensitivity is $M A E$ duration. We therefore also recorded the MAE duration for the moving component under transparent conditions with one stationary and one moving pattern. Some reasons for using MAE duration are discussed in Burke and Wenderoth (1993). Other psychophysical correlates like MAE speed would also have been a possibility.

\footnotetext{
* Recently a new method for measuring the strength of MAEs has been presented by Lankheet, Verstraten and Møller (1993) and Blake, Steiner and Rose (1993).
}

However, like MAE duration, MAE speed measurement has its problems as well, e.g. Anstis (1986) called magnitude estimation a "method of last resort", whereas Sekuler and Pantle (1967) called the method of nulling "obtrusive".*

\section{Methods and procedure}

Directional motion sensitivity measurements. In this experiment one of the RPA was stationary and the other RPA was moving either vertically upwards to the right or vertically upwards to the left (both deviating $45 \mathrm{deg}$ from the $y$-axis). There is no qualitative perceptual difference regarding the transparency phenomenon between this case and the one where both patterns have non-zero velocity. Threshold determination was done by a two-alternative forced-choice discrimination. Subjects had to indicate in which direction the movement was perceived, for example from bottom-right to top-left corner or vice versa (in the brtl condition).

Sensitivity was measured by using a luminance signal to noise ratio (LSNR) paradigm. The luminance of the moving pattern was modulated by a noise pattern. The root mean square (r.m.s.) contrast of the moving RPA is $r_{\mathrm{s}}$ and that of the noise pattern (completely refreshed with every new frame) $r_{\mathrm{n}}$. The subject or the experimenter manipulated or set the LSNR $=r_{3}^{2} / r_{n}^{2}$ while the total contrast $C=\left(r_{\mathrm{n}}^{2}+r_{\mathrm{s}}^{2}\right)^{1 / 2}$ was automatically kept constant. This was accomplished via an electronic lookup table in the stimulus control hardware. The basis of this method has previously been described by van Doorn and Koenderink (1982a). For a detailed discussion of this LSNR method, see the Appendix of Fredericksen et al. (1993). The LSNR of the stationary pattern was held at a constant suprathreshold value, 200, during the whole experiment. It was found in pilot experiments that this choice is not at all critical and at this LSNR value no "noise" is perceived for this pattern. The contrast level was $70 \%$, which is well above the $35 \%$ level where the motion SNR threshold becomes independent of contrast (van de Grind, Koenderink \& van Doorn, 1987). The thresholds were determined with a staircase procedure that tracked a $79 \%$ correct level; three consecutive correct directional discriminations resulted in a lowering of the LSNR by $2 \mathrm{~dB}$. If a mistake was made the LSNR went up by the same amount. This was done for 10 reversals. The LSNR value was calculated by averaging the last six reversals. After every session the reversals were inspected. If the sequence did not reach a stable threshold value, it was remeasured. This happened for fewer than $3 \%$ of all staircases.

A single session consisted of a stationary pattern combined with a moving pattern of one of the following 13 speeds $0.25,0.5,1,2,4,6,8,10,12,16,20,24$, and $28 \mathrm{deg} \mathrm{sec}^{-1}$. A session lasted about $35 \mathrm{~min}$. Speeds were pseudo-randomized. Subjects could rest at any time during the experiment. In case a stimulus was missed a new stimulus could be brought up again by pressing a key on the keyboard. In order to decrease the required duration of the experiment and to minimize the influence 
of adaptation, the subjects manipulated the initial value of the LSNR manually until it was close to but still clearly above perceptual threshold. As soon as the initial LSNR-value was set the experiment began. Subjects were presented with the test stimulus for $1.0 \mathrm{sec}$.

In order to compensate for possible directional anisotropies the motion sensitivity curve was measured for both motion in the bottom-right/top-left (br $\leftrightarrow \mathrm{tl}$ ) and in the orthogonal bottom-left/top-right (bl $\leftrightarrow \mathrm{tr}$ ) direction along the diagonal orientations. A square display window of $4 \mathrm{deg}$ was used. All curves were measured twice on different days.

Measurements of motion aftereffect duration. In this part of the second experiment we again combined a stationary pattern with a moving pattern. Not all speeds were used since it proved in pilot runs that for this kind of stimulus speeds above $14 \mathrm{deg} \mathrm{sec}-1$ did not give any MAEs at all. The speeds used were $0.25,0.50,1,2,4,6$, $8,10,12$, and $14 \mathrm{deg} \mathrm{sec}^{-1}$. Both the stationary and the moving pattern were set at a LSNR of 200. Display size was $4 \mathrm{deg}$ again. The subjects adapted to the stimulus for $60 \mathrm{sec}$. After $60 \mathrm{sec}$ the moving pattern was stopped and the duration of the MAE was recorded by using a stopwatch. The inter-trial interval was $90 \mathrm{sec}$. Two curves were measured: the MAE duration of motion from the bottom-left to top-right $(\mathrm{bl} \rightarrow \mathrm{tr})$ and for motion from the bottom-right to top-left $(\mathrm{br} \rightarrow \mathrm{tl})$. All curves were repeated three times on different days and the speeds were pseudo randomized.

\section{Results and discussion}

Directional motion sensitivity. Figure 2 shows results of the directional motion sensitivity experiment for all subjects. All subjects are most sensitive in the range from 1 to $8 \mathrm{deg} \mathrm{sec}^{-1}$. For subjects FV and WD the differences between results for motion along the two axes $(\mathrm{bl} \leftrightarrow \operatorname{tr}$ vs $b r \leftrightarrow t$ l) are minimal, for subject $\mathrm{JH}$ there is a difference. $\mathrm{JH}$ is more sensitive to a direction along the bottom-left $\leftrightarrow$ top-right axis (open squares) than for motion along the orthogonal axis (solid squares). We will use the results of Fig. 2 later on to predict the direction of the MAE of transparent motion (section predictions).

$M A E$ duration. The results of the MAE duration experiment for all subjects are shown in Fig. 3. There are large differences between subjects as far as total duration is concerned. The MAEs of subject WD lasted much longer than those of JH and the durations for $\mathrm{FV}$ are intermediate. The difference within subjects between directions, however, is rather small, especially in view of the fact that it is difficult to measure MAE duration and that small shifts in criterion are believed to result in large shifts in duration (see Thompson, 1976). The results of Fig. 3 will be used in the next section to develop a prediction for the direction of the MAE of transparent motion.

It should be noted that the perception of a MAE is not related to motion sensitivity in a simple or obvious manner. For example, subject WD is least sensitive to a pattern moving with a speed of $0.25 \mathrm{deg} \mathrm{sec}^{-1}$. However

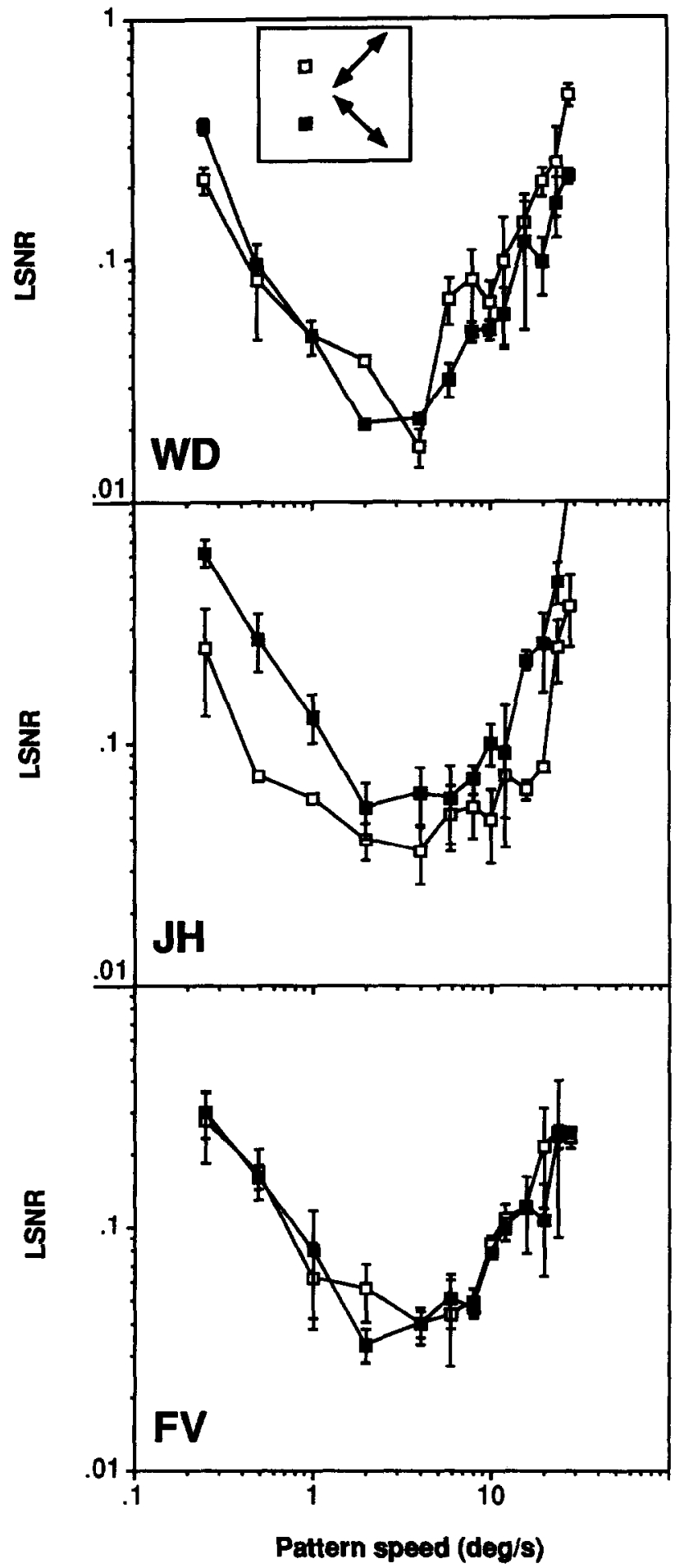

FIGURE 2. Motion sensitivity curves for speeds $(0.25,0.5,1,2,4,6$, $8,10,12,16,20,24$, and $28 \mathrm{deg} \mathrm{sec}^{-1}$ ). The open symbols represent the directional sensitivity on the bottom-left to top-right axis and vice versa (bl $\leftrightarrow \mathrm{tr}$ ). The solid symbols for the bottom-right to top-left axis and vice versa $(\mathrm{br} \leftrightarrow \mathrm{tl})$. The bars represent $\mathrm{ISD}$.

his MAE duration for that speed nearly reaches an average of $10 \mathrm{sec}$, and though WD is more sensitive to a speed of $14 \mathrm{deg} \mathrm{sec}^{-1}$ he does not perceive a MAE at all at this speed. It seems that the perceptual threshold for the perception of a MAE is not determined in a simple straightforward way by motion sensitivity. 
Furthermore, subjects WD and FV have about the same thresholds for $0.25 \mathrm{deg} \mathrm{sec}^{-1}$ but $\mathrm{FV}$ does not perceive a MAE for this speed, whereas WD does.

\section{PREDICTIONS}

It was concluded from Expt 1 that formula (1) with $c_{1}$ and $c_{2}$ taken as constants cannot account for the MAE

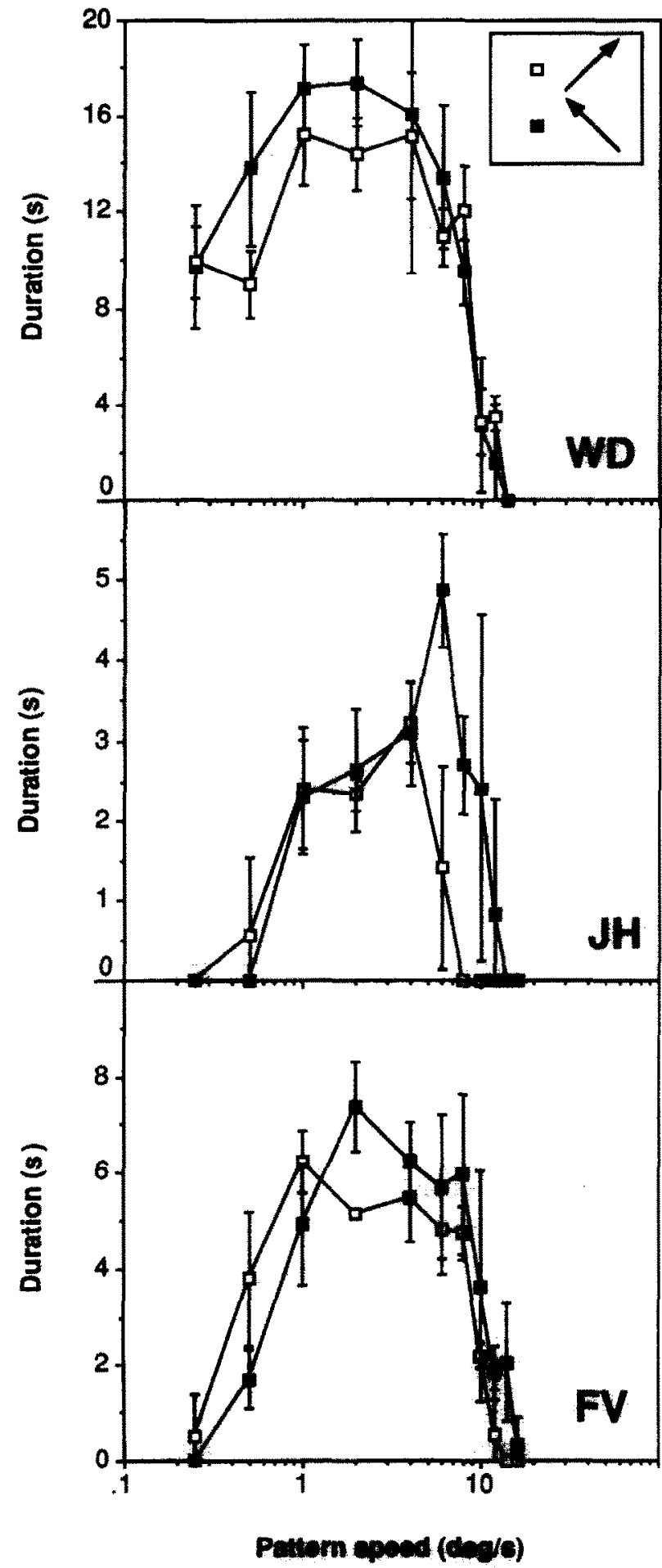

FIGURE 3. MAE duration after transparent conditions for speeds $\left(0.25,0.5,1,2,4,6,8,10,12,14\right.$, and $\left.16 \mathrm{deg} \mathrm{sec}^{-1}\right)$. The open symbols represent the MAE duration in the bl $\rightarrow \mathrm{tr}$ (bottom-left $\rightarrow$ top-right) condition. The solid symbols represent the br $\rightarrow \mathrm{tl}$ condition. The bars represent $1 \mathrm{SD}$. direction of transparent motion. This implies that formula (1) needs to be revised to cover the general case. We suggested above to take sensitivity differences into account. Let us assume that the LSNR-threshold value for a movement vector $\mathbf{V}$ is $\operatorname{LSNR}(\mathbf{V})$ and let us define the sensitivity $S(V)$ as the inverse of this threshold. Then an "external" vector $\mathbf{V}$ may be assumed to give rise to a neural or internal vector

$$
\mathbf{V}_{\mathrm{n}}=c S(\mathbf{V}) \mathbf{U}_{\mathrm{v}}
$$

where $U_{v}$ is the unit vector in the direction of $\mathbf{V}$ or $\mathbf{U}=\mathbf{V} /|\mathbf{V}|$. Thus neural vector $\mathbf{V}_{\mathrm{n}}$ represents the direction of $\mathbf{V}$ and has a magnitude of $c$ times the sensitivity to $\mathbf{V}$. If we combine formula (2) with formula (1) and denote the unit vectors in the directions of $V_{1}$ and $V_{2}$ as $U_{1}$ and $\mathrm{U}_{2}$ respectively we get

$$
V_{\mathrm{MAE}}=c S\left(\mathbf{V}_{1}\right) \mathbf{U}_{1}+c S\left(\mathbf{V}_{2}\right) \mathbf{U}_{2} .
$$

In fact we only measured the direction of the MAE as a function of $V_{1}$ and $V_{2}$ for fixed perpendicular directions of the adaptation velocities. The speed of the MAE was not measured, so formula (3) only needs to predict the direction of the compound MAE. With the convention of Fig. 1 (see panel 14) we then find

$$
\alpha_{\text {MAE }}=225 \mathrm{deg}+\arctan \left(\frac{S\left(\mathbf{V}_{2}\right)}{S\left(\mathbf{V}_{1}\right)}\right) .
$$

For the direction of the MAE of transparent motion based on the MAE duration (dur) of its individual vectors we similarly get

$$
\alpha_{\mathrm{MAE}}=225 \mathrm{deg}+\arctan \left(\frac{\operatorname{dur}\left(\mathbf{V}_{2}\right)}{\operatorname{dur}\left(\mathbf{V}_{1}\right)}\right) .
$$

Figures 4 and 5 show the calculated directions on the basis of sensitivity [formula (4), solid circles] and MAE duration [formula (5), open squares] together with the empirical data (no symbol) of Expt 1.

For subject FV all possible combinations are shown (Fig. 4). For subject WD (Fig. 5, upper four panels) four values for $V_{2}$ are combined with all speeds for vector $V_{1}$. The same is true for subject JH (Fig. 5, lower four panels). Missing data points were either not perceived (empirical condition) or were not calculated (in the case of the predictions). For example in the first graph of JH the predicted directions on the basis of MAE duration are not displayed, simply because JH did not perceive a MAE for this magnitude of vector $V_{2}$ (see Fig. 3). The predicted directions approach the empirical values reasonably well, especially in the most sensitive range.

Statistical analyses using a "goodness of fit" procedure revealed differences between predictions based on MAE duration and directional sensitivity. The curves were compared in pairs using the variances between the raw data and the fitted curves (fitted by a second-order polynom). The test statistic was dependent on the total number of observations (either empirical or calculated). In general we used $F_{3,14}=3.34$ where $\alpha=0.05$. 


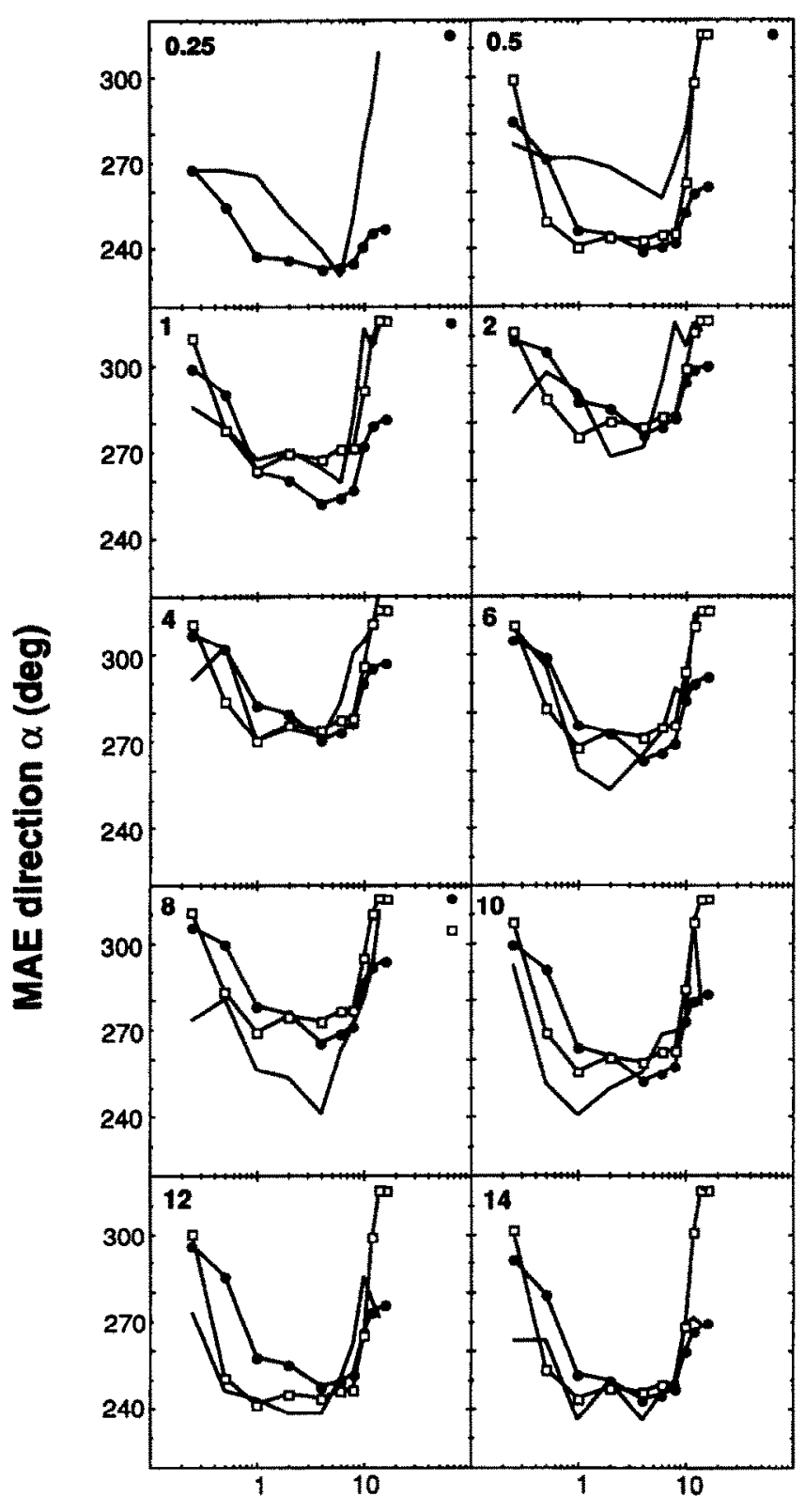

Pattern Speed (deg/s)

FIGURE 4. Empirical and predicted directions of transparent motion for subject $\mathrm{FV}$ and all speed combinations. For ease of distinction the panels present the direction of the MAE as a function of $V_{2}$ with $V_{1}$ as a parameter. MAE direction is given in degrees according to the convention in panel "14" of Fig. 1. The solid line without symbols represents the empirical directions as measured in Expt 1. Solid circles are the directions predicted on the basis of the LSNR values as measured in Expt 2 (see Fig. 2). Open squares represent the predicted directions on the basis of MAE duration as presented in Fig. 3. Where there are symbols missing the direction was either not perceived or could not be calculated because of missing values. For example subject FV did not perceive a MAE for $V_{2}=0.25 \mathrm{deg} \mathrm{sec}^{-1}$ (see Fig. 3). In that case the predicted values could not be calculated and therefore the corresponding curve is missing. For the same reason some values are missing for the other subjects (see Fig. 5). Since the LSNR threshold value was not measured for $14 \mathrm{deg} \mathrm{sec}^{-1}$ we used the LSNR value of $16 \mathrm{deg} \mathrm{sec}^{-1}$. A solid circle or an open square in the right corner of the panels implies that the corresponding curve differed significantly (as tested by a goodness of fit test) from the empirical curve. For subject FV sensitivity-based predictions were wrong for $0.25,0.5,1$ and $8 \mathrm{deg} \mathrm{sec}^{-1}$, whereas duration based predictions were wrong only for the $8 \mathrm{deg} \mathrm{sec}-1$ condition.

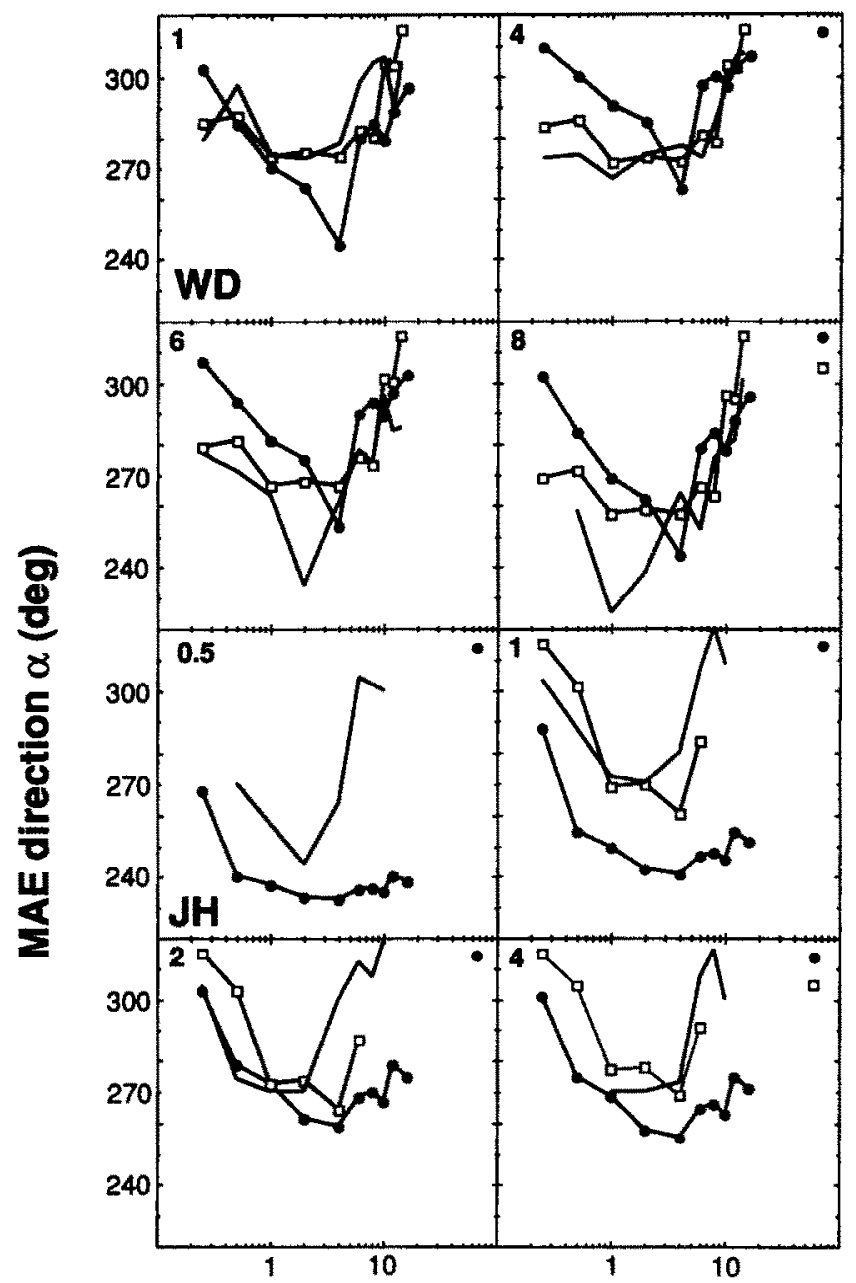

Pattern Speed (deg/s)

FIGURE 5. Results for subject WD (upper four panels) and subject JH (lower four panels) for four values of $V_{2}$. See for further explanation the caption of Fig. 4.

Results of this analyses show that three out of 16 curves as predicted by the MAE duration data differed significantly from the empirical curves (shown as open squares in the upper-right corner of the panels of Figs 4 and 5). For sensitivity curves this number was considerably higher, 10 out of 18 curves differed significantly from the empirical curves (shown as solid circles in the upperright corner of the panels of Fig. 5). It can therefore be concluded that formula (5), based on the MAE duration of the individual vectors, is a better predictor of the MAE direction of transparent motion than formula (4), based on sensitivity. The fact that directional sensitivity predicts different results may be related to an earlier observation (see section results) that perceiving a MAE is not strictly related to directional sensitivity. It could well be that the MAE duration and directional sensitivity are parameters set in different pathways. This is not an unreasonable assumption since a two-dimensional moving pattern has two directions while seen directly whereas the MAE is uni-dimensional. In the first case separate groups of directionally sensitive units are apparently involved, in the latter case they may not, since we then only have perceptual evidence of one compound effect. 


\section{GENERAL DISCUSSION}

We have investigated the direction of the MAE that results from transparent motion. The results show that the MAE of transparent motion is unidirectional and was not seen to change its direction while present. The direction of this MAE is determined by some vector addition weighted according to formulas (4) or (5) by the amount of adaptation induced by the individual adaptation vectors. Within a restricted range MAE duration of the individual vectors is a good weighting factor for the vector summation [formula (5)] predicting the MAE direction of transparent motion. Formula (5) predicts the empirical results better than formula (4) which was based on the directional sensitivity values.

Are these results compatible with existing models of motion detection and the two models purporting to explain MAEs as summarized in the Introduction? Most of these motion detection models can be translated into some form of "elaborated Reichardt detectors" (Van Santen \& Sperling, 1984, 1985) and such a bidirectional detector cannot explain transparent motion (at least not for oppositely directed motion components, which gives perfect transparency). Therefore, it has been assumed by van Doorn et al. (1984) that the two uni-directional "subunits" of the Reichardt model, extended with front-end receptive fields (spatio-temporal filters), feed independently into the cooperation stage. These subunits were aptly called "bi-local" detectors, since they consist of two spatially non-coinciding receptive fields, the inter-centre distance of which was called the span. The bi-local detector performs what Sperling, Van Santen and Burt (1985) call a "delay-and-compare" operation. Any image element that travels the span in the detector-specific delay time leads to a report of motion, with a confidence tag (the detector's firing rate), to the cooperation stage. A model of this kind allows the motion transparency percept. To explain MAEs the automatic gain control of the classic model should then be placed at or after the cooperation stage. The "classic" explanation of the MAE, as formulated by Sutherland (1961) cannot help us to explain the MAE of transparent motion. Sutherland's model also assumes a balance between oppositely directed motion detectors and places the adaptation process (gain control) before the balance, at the individual motion detectors. If the model were modified to allow the perception of transparency of oppositely directed motion, the latter assumption would still be wrong or inconvenient. The difference between primary percept (two motion vectors) and the MAE (one vector) would still mean that gain control/adaptation at individual motion detectors cannot suffice. Some vector operation on ensembles of detectors is seen from our results to be a minimum requirement of any model of this MAE.

Let us now turn to the distribution shift model as formulated by Mather (1980), keeping the restriction in mind that the front-end detectors have to be unilateral to allow transparency. Let us use the above data to show a discrepancy between expectations based on his model and our findings.

Suppose two motion components described by orthogonal vectors with different lengths (speeds) are combined under transparent conditions. Moreover, suppose that the MAEs of the individual components have different durations. This implies that the time to recover from adaptation is different for the two components. Since the two component MAEs have different durations the contribution of the component inducing the shortest duration aftereffect will not last as long as that of the component inducing the longer MAE duration. It is therefore expected that the direction of the MAE of transparent motion will eventually end up in the opposite direction of the vector with the longest duration. The distribution shift model, if it is assumed to describe an ongoing rather than a sample-and-hold process, would predict that the direction of the integrated MAE would have to change during the recovery from adaptation. Starting from the direction of the vector sum it would have to change towards a direction opposite the component with the longest MAE duration (so towards either 225 or $315 \mathrm{deg}$ ). Our observations and subject reports are incompatible with this prediction. Nobody has observed such shifts of directions during these experiments. It might be objected that we have not explicitly measured the stability of the MAE direction. Due to the inherent low resolution of the MAEs, small changes in MAE direction may not be detectable. On the other hand, changes toward the opposite of one of both inducing vectors would have been clearly visible. Moreover, the good continuation of the empirical curves as presented in Fig. 1 show that MAE direction can be recorded rather accurately. Our results thus appear to speak against the distribution shift model, at least in its present form.

So far, it seems that as long as the MAE is experienced, its direction does not change over time. The distribution shift model has many positive aspects and it might therefore be worthwhile to modify it in such a way that it can also cover the case of a MAE of transparent motion. This requires that the assumption that individual motion detectors adapt should be dropped. The adaptation process, presumably revealing its character in the MAE, has to take place at or after the cooperation stage. Moreover, it does not slavishly follow the signals responsible for the percept of transparency during adaptation. It performs some sort of separate "integration", described above by the weighted vector summation.

\section{The possible neural site of the MAE of transparent motion}

The MAE of transparent motion seems to be an exponent of an integrative process. Physiological research so far has indicated that the integrative aspects of motion perception take place at a level central to area V1. Snowden, Treue, Erickson and Andersen (1991) have shown in rhesus monkeys that direction selective 
cells in area V1 respond to their preferred direction under both non transparent and transparent conditions, with only minimal differences. In other words VI cells behave like directional filters in both conditions. Cells in area MT, however, show clear differences in activity under transparent and non transparent motion conditions. These MT cells show suppression of their activity under transparent conditions. Snowden et al. suggested that this suppression of MT cells may reflect interactions between different motion directions. Area MT thus seems to be involved in some aspects of the integration of motion information. Presentation times that were used by Snowden and his colleagues were too short to adapt the cells. Although some electrophysiological research on aftereffects has been reported (e.g. Hammond, Mouat \& Smith, 1986), to our knowledge no electrophysiological studies have focused on the MAE of transparent motion. In future electrophysiological research it would be interesting to investigate whether the suppression of MT cells shows an "aftereffect" when the motion has stopped. Furthermore, it would have to be shown that the activity distribution in MT cells can be manipulated by changing the individual vectors of the inducing stimulus.

Although several reports have suggested area MT as the location where the MAE of bi-vectorial motion arises (e.g. Wenderoth, Bray \& Johnstone, 1988), there are considerations that make it tempting to suggest that the MAE stems from a different central part of the brain. It has recently been suggested (e.g. Chaudhuri, 1991) that MAEs may stem from parts of the brain where motor signals (eye and or head motion) are explicitly taken into account. We performed some experiments using transparent motion and pursuit eye movements that also suggest a different locus than V1 or MT. We found that if one of the vectors is pursued, the direction of the MAE of transparent motion is determined both by retinal stimulation and by efference copy signals. Eye movements influence the direction of the MAE of transparent motion and it was concluded from our results [to be reported in detail elsewhere (for a summary see Grüsser, Fredericksen, van de Grind \& Verstraten, 1992)] that the MAE is probably generated at a locus where gaze movement signals interact with retinal motion signals. Area MST and FST are possible candidates. Of course the vectors of transparent motion can be integrated at, say, the level of MT and the information from this level might then interact with other neural responses giving rise to a different percept than expected from the properties of MT alone. Transparent motion is perceived as two superimposed moving patterns. This means that both vectors retain this directional information along the whole way from retinal stimulation to percept. The motion components are not integrated to global "averaged" motion along the way to perception, which might in view of the mentioned finding by Snowden et al. (1991) call the role of MT even in this type of transparent motion perception into question. In any case, transparent motion and its MAE pose an interesting new challenge for both psychophysics and electrophysiology.

\section{REFERENCES}

Anstis, S. M. (1986). Motion perception in the frontal plane: Sensory aspects. In Boff, K. R., Kaufman, L. \& Thomas, J. P. (Eds), Handbook of perception and human performance. New York: Wiley.

Barlow, H. B. \& Hill, R. M. (1963). Evidence for a physiological explanation of the waterfall illusion and figural aftereffects. Nature, $200,1434-1435$.

Blake, R., Steiner, V. \& Rose, D. (1993). Aftereffects of adaptation to different types of motion. Investigative Ophthalmology and Visual Science (Suppl.), 34, 1363.

Burke, D. \& Wenderoth, P. (1993). Determinants of two-dimensional motion aftereffects induced by simultaneously- and alternately presented plaid components. Vision Research, 33, 351-359.

Chaudhuri, A. (1991). Eye movements and the motion aftereffect: Alternatives of the induced motion hypothesis. Vision Research, 31 , 1639-1645.

Clarke, P. G. H. (1977). Subjective standstill caused by the interaction of moving patterns. Vision Research, 17, 1243.

van Doorn, A. J. \& Koenderink, J. J. (1982a). Temporal properties of the visual detectability of moving spatial white noise. Experimental Brain Research, 45, 179-188.

van Doorn, A. J. \& Koenderink, J. J. (1982b). Spatial properties of the visual detectability of moving spatial white noise. Experimental Brain Research, 45, 189-195.

van Doorn, A. J. \& Koenderink, J. J. (1983). Detectability of velocity gradients in moving random-dot patterns. Vision Reserch, 23, 799-804.

van Doorn, A. J., Koenderink, J. J. \& van de Grind, W. A. (1984) Limits in spatio-temporal correlation and the perception of visual movement. In van Doorn, A. J., van de Grind, W. A. \& Koenderink, J. J. (Eds), Limits in perception, essays in honour of Maarten A. Bouman. Utrecht: VNU Science Press.

van Doorn, A. J., Koenderink, J. J. \& van de Grind, W. A. (1985) Perception of movement and correlation in stroboscopically presented noise patterns. Perception, 14, 209-224.

Fredericksen, R. E., Verstraten, F. A. J. \& van de Grind, W. A. (1993) Spatio-temporal characteristics of human motion perception. Vision Reseurch, 33, 1193-1205.

van de Grind, W. A., Koenderink, J. J. \& van Doorn, A. J. (1987). Influence of contrast on foveal and peripheral detection of coherent motion in moving random-dot patterns. Journal of the Optical Society of America A, 4, 1643-1652.

Grüsser, O.-J., Fredericksen, R. E., van de Grind, W. A. \& Verstraten, F. A. J. (1992) Spatial summation of movement aftereffects and its modification by eye pursuit movements. Society of Neuroscience Abstracts, 18, 1034.

Hammond, P., Mouat, G. S. V. \& Smith, A. T. (1986). Motion after-effects in cat striate cortex elicited by moving texture. Vision Research, 26, 1055-1060.

Lankheet, M. J. M., Verstraten, F. A. J. \& Møller, P. (1993). Selective attention to the components of transparent motion stimuli modulates the motion aftereffect. Investigative Ophthalmology and Visual Science (Suppl.), 34, 1363.

Mather, G. (1980). The movement aftereffect and a distribution-shift model for coding the direction of visual movement. Perception, 9 , 379-392.

Mather, G. \& Moulden, B. (1980). A simultaneous shift in apparent direction: Further evidence for a "distribution-shift" model of dirfection coding. Quarterly Journal of Experimental Psychology, 32, 325-333.

Richards, W. (1971). Motion detection in man and other animals. Brain Behavior and Evolution, 4, 162-181.

Sekuler, R. \& Pantle, A. (1967). A model for the aftereffects of seen movement. Vision Research, 7, 427-439.

Snowden, R. J., Treue, S., Erickson, R. G. \& Andersen, R. A. (1991). The response of area MT and V1 neurons to transparent motion. Journal of Neuroscience, 11, 2768-2785.

Sperling, G., Van Santen, J. P. H. \& Burt, P. J. (1985). Three theories of stroboscopic motion detection. Spatial Vision, l, 47-56.

Sutherland, N. S. (1961). Figural aftereffects and apparent size. Quarterly Journal of Experimental Psychology, 13, 222-228. 
Thompson, P. G. (1976). Velocity after-effects and the perception of movement. Ph.D. dissertation, University of Cambridge, Cambridge, England.

Van Santen, J. P. H. \& Sperling, G. (1984). Temporal covariance model of human motion perception. Journal of the Optical Society of America A, 1, 451-473.

Van Santen, J. P. H. \& Sperling, G. (1985). Elaborated Reichardt detectors. Journal of the Optical Society of America A, 2, 300-321.

Wenderoth, P., Bray, R. \& Johnstone, S. (1988). Psychophysical evidence for an extra-striate contribution to a pattern-selective motion aftereffect. Perception, 17, 81-91.

Wohlgemuth, A. (1911). On the aftereffect of seen movement. British Journal of Psychology Monographs (Suppl. I).
Acknowledgements-Frans Verstraten is supported by the Biophysics Foundation of the Netherlands Organization for Scientific Research (NWO) and Eric Fredericksen was supported by a UNC Cognitive Science Program Pre-doctoral Fellowship, and an Inernational Brain Research Organization Research Fellowship. We are grateful to Jane Boulton, O.-J. Grüsser, Andrea Koenderinkvan Doorn, Martin Lankheet and Peter Wenderoth for constructive comments on the experiments and earlier drafts of this paper. We also thank Wim van Damme and Johan Hulleman for participating in the experiments and the electronic engineers and hardware designers Pieter Schiphorst and Rob van Weerden for their assistance. 